\title{
Ginsenoside Rd promotes glutamate clearance by up-regulating glial glutamate transporter GLT-1 via PI3K/AKT and ERK1/2 pathways
}

\author{
Xiao Zhang ${ }^{1}$, Ming Shi ${ }^{1}$, Magnar Bjørås ${ }^{2}$,Wei Wang ${ }^{2}$, Guangyun Zhang ${ }^{1}$, Junliang Han ${ }^{1}$, Zhirong Liu ${ }^{1}$, \\ Yunxia Zhang ${ }^{1}$, Bing Wang ${ }^{1}$, Jing Chen ${ }^{1}$, Yi Zhu ${ }^{1}$, Lize Xiong ${ }^{3}$ and Gang Zhao ${ }^{1}{ }^{*}$ \\ ' Department of Neurology, Xijing Hospital, The Forth Military Medical University, Xi'an, Shaanxi, China \\ ${ }^{2}$ Department of Microbiology, Institute of Clinical Medicine, Oslo University Hospital, Oslo, Norway \\ ${ }^{3}$ Department of Anesthesiology, Xijing Hospital, The Forth Military Medical University, Xi'an, Shaanxi, China
}

\section{Edited by:}

Morris Karmazyn, University of

Western Ontario, Canada

Reviewed by:

Bin-Nan Wu, Kaohsiung Medical University, Taiwan

Morris Karmazyn, University of Western Ontario, Canada

${ }^{*}$ Correspondence:

Gang Zhao, Department of Neurology, Xijing Hospital, The Forth Military Medical University, 15 Changle-xi Road, Xi'an, Shaanxi 710032, China e-mail: zhaogang@fmmu.edu.cn
Ginsenoside $\mathrm{Rd}(\mathrm{Rd})$, one of the main active ingredients in Panax ginseng, has been showed to protect against ischemic cerebral damage both in vitro and in vivo. However, the underlying mechanism of $\mathrm{Rd}$ is largely unknown. Excessive extracellular glutamate causes excitatory toxicity, leading to cell death, and neurodegenerative processes after brain ischemia. The clearance of extracellular glutamate by astrocytic glutamate transporter GLT-1 is essential for neuronal survival after stroke. Here we investigated the effects of $\mathrm{Rd}$ on the levels of extracellular glutamate and the expression of GLT-1 in vivo and in vitro. After rat middle cerebral artery occlusion, Rd significantly increased the mRNA and protein expression levels of GLT-1, and reduced the burst of glutamate as revealed by microdialysis. Consistently, specific glutamate uptake by cultured astrocytes was elevated after Rd exposure. Furthermore, we showed that Rd increased the levels of phosphorylated protein kinase $B$ (PKB/Akt) and phospho-ERK1/2 (p-ERK1/2) in astrocyte culture after oxygen-glucose deprivation. Moreover, the effect of Rd on GLT-1 expression and glutamate uptake can be abolished by PI3K/AKT agonist LY294002 or ERK1/2 inhibitor PD98059. Taken together, our findings provide the first evidence that $\mathrm{Rd}$ can promote glutamate clearance by up-regulating GLT-1 expression through PI3K/AKT and ERK1/2 pathways.

Keywords: ginsenoside Rd, GLT-1, astrocyte, glutamate, PI3K/AKT, ERK1/2

\section{INTRODUCTION}

In spite of advances in technology and improved clinical care, ischemic stroke still remains a major cause of mortality and disability in human and the second leading cause of death in developed countries (Goldstein et al., 2006). Two major approaches have been developed to treat acute ischemic stroke: recanalization and neuroprotection (Gropen et al., 2006). The goal of neuroprotective therapy is to save potentially viable brain tissue in the ischemic penumbra. Unfortunately, although many neuroprotective interventions are effective for stroke in the animal model, they failed to benefit to patients in clinical trials. Thus, developing effective drugs to treat acute ischemic stroke still faces challenges.

Ginseng, the root of Panax ginseng C. A. Meyer (Araliaceae), has been widely used as a kind of traditional Chinese herbal medicine for 1000s of years. Ginsenosides are the most active ingredients in ginseng. Up to now more than 40 different ginsenosides, including ginsenoside Rd (Rd) have been identified (Radad et al., 2006). Our randomized, double-blind, placebo-controlled, multicenter trial showed that $\mathrm{Rd}$ is effective and safe for the treatment of acute ischemic stroke (Liu et al., 2009, 2012b).

In our pre-clinical studies, we found that Rd can prevent glutamate/oxygen-glucose deprivation (OGD)-induced apoptosis in cultured neurons (Ye et al., 2009; Li etal., 2010), and reduce infarction volume after transient focal ischemia in rats (Ye et al., 2011a,b), suggesting that Rd can be served as a promising neuroprotectant. However, the underlying mechanisms of Rd neuroprotection are still not fully elucidated. Numerous approaches in neuroprotection have considered the application aimed at targeting non-neuronal cells (Van der Schyf et al., 2006). Besides directly supporting neurons, Rd can interfere with a number of other cells such as astrocytes (Lopez et al., 2007). Astrocytes play an important role in supporting neurons in physiological and pathological conditions by producing various growth factors. Particularly, they are the key cells for the uptake of excitatory neurotransmitter glutamate (Danbolt, 2001). Excessive extracellular glutamate elicits neurotoxicity and is mainly removed by glutamate transporters, GLAST (EAAT1) and GLT-1(EAAT2), exclusively expressed on astrocytes (Gegelashvili and Schousboe, 1997). GLAST predominantly expresses in the cerebellum and GLT-1 in the cerebral cortex and hippocampus (Danbolt, 2001). In the forebrain, more than $90 \%$ of the glutamate uptake is mediated by GLT-1(Danbolt, 2001), and dysfunction or knockout of GLT-1 gene leaded to elevation of extracellular glutamate and exacerbation of acute cortical injury (Rao et al., 2001; Mitani and Tanaka, 2003). Studies showed that GLT-1 expression was regulated by PI3K/AKT and ERK1/2 pathways (Li et al., 2006; Lee et al., 2012a) and ginsenosides can activate these pathways (Lan et al., 2011; Hashimoto et al., 2012; Yan et al., 2013). Therefore, 
in this study we investigated the effects of Rd on extracellular glutamate metabolism and the expression of GLT-1, and further explored whether PI3K/AKT and ERK1/2 pathways were involved in this process.

\section{MATERIALS AND METHODS MATERIALS}

Rd with a purity of $98 \%$ was obtained from Tai-He Biopharmaceutical Co. Ltd. (Guangzhou, China). The stock solutions were prepared in saline containing 10\% 1,3-propanediol $(\mathrm{v} / \mathrm{v})$. Hoechst 33342 was purchased from Sigma-Aldrich Inc. (St. Louis, MO, USA). The commercial kit for the detection of glutamate was purchased from CMA (Solna, Sweden). GLT-1 antibody was obtained from Abcam (Cambridge, UK) and other antibodies were purchased from Cell Signaling (Danvers, MA, USA). All other reagents were from commercial suppliers and of standard biochemical quality.

\section{FOCAL CEREBRAL ISCHEMIA}

Male Sprague-Dawley rats weigh 270-320 g were used in this study. Animal protocols were approved by the Ethics Committee for Animal Experimentation of the Fourth Military Medical University. The focal cerebral ischemia was induced by $1.5 \mathrm{~h}$ of middle cerebral artery occlusion (MCAO) as described previously with modifications (Kramer et al., 2010). In brief, animals were anesthetized with a mixture of isoflurane (1.5-2\%), oxygen and nitrogen. Body temperature in the rectum was maintained at $37^{\circ} \mathrm{C}$ using a thermostatically controlled heating blanket connected to a thermometer probe. A 4-0 nylon monofilament coated with poly-L-lysine was introduced through the internal carotid artery to occlude the origin of the middle cerebral artery (MCA). The induction of focal cerebral ischemia was verified with laser Doppler flowmetry (PeriFlux 5000; Perimed AB, Sweden). A drop in regional cerebral blood flow (CBF) below $30 \%$ from baseline after the insertion of the filament was considered to be sufficient for induction of focal cerebral ischemia. Control animals were subjected to the same surgical procedures except that the suture was not advanced into the MCA. Rd with a concentration of $30 \mathrm{mg} / \mathrm{kg}$ or vehicle was applied intraperitoneally $1 \mathrm{~h}$ before MCAO. Animals were sacrificed at designated times points after MCAO and samples were collected for Western blot and reverse transcription polymerase chain reaction (RT-PCR) experiments analysis. The tissues from the ischemic core and penumbra were dissected according to the protocol described by Kramer et al. (2010).

\section{ASTROCYTE CULTURES}

Astrocytes were cultured from newborn Sprague-Dawley rats as previously described with some modifications (Lopez et al., 2007). Rat cortex and striatum were isolated and minced. After trypsinization, dissociated cells were passed through sterile nylon meshes, seeded to $75 \mathrm{~cm}^{2}$ flask at a density of $1 \times 10^{6}$ cells and cultured in Dulbecco's-modified eagle medium (DMEM, GIBCO) supplemented with $10 \%$ heat-inactivated fetal bovine serum at $37^{\circ} \mathrm{C}$. The medium was renewed three times a week. Microglia and oligodendrocytes were removed by shaking at $260 \mathrm{pm}$ overnight at $37^{\circ} \mathrm{C}$ in an orbital shaker (ShenTong, China). Astrocytes were collected and sub-cultured for $24 \mathrm{~h}$ before experiments.

\section{OXYGEN-GLUCOSE DEPRIVATION}

Oxygen-glucose deprivation was carried out as described by $\mathrm{Li}$ etal. (2010). Briefly, the culture medium was replaced with pre-warmed DMEM without glucose and serum. The cell cultures were then transferred into an anaerobic chamber equilibrated with $95 \% \mathrm{~N}_{2}$ and $5 \% \mathrm{CO}_{2}$. The chamber was kept in a $37^{\circ} \mathrm{C}$ incubator. Control cultures were maintained in a normal oxygenated DMEM containing $25 \mathrm{mM}$ glucose. After $3 \mathrm{~h}$, cultures were placed back to the normoxic incubator with normal culture medium.

\section{MICRODIALYSIS}

Microdialysis was performed as described previously (Ye et al., 2011b). Briefly, a microdialysis probe (BAS MD-2204, 4 mm membrane) was stereotaxically inserted into the right striatum through the cannula guide $(-3 \mathrm{~mm}$ anteroposterior, $+4 \mathrm{~mm}$ mediolateral to the Bregma, $-4 \mathrm{~mm}$ dorsoventral from the surface of the brain). Artificial cerebrospinal fluid ( $\mathrm{NaCl} 135 \mathrm{mM}, \mathrm{KCl} 1 \mathrm{mM}$, $\mathrm{CaCl}_{2} 1.2 \mathrm{mM}, \mathrm{MgCl}_{2} 1 \mathrm{mM}, \mathrm{pH} 7.4$ ) was perfused at $2 \mu \mathrm{l} / \mathrm{min}$ using a microinjection pump (BeeHive BAS, USA). The microdialysis samples were continuously collected into microvials collected every $20 \mathrm{~min}$. Three samples were collected as baseline values at the end of $2 \mathrm{~h}$ equilibration period. The concentrations of glutamate in the microdialysis samples were determined using a CMA 600 Analyzer (Solna, Sweden). Level changes for all measured chemicals were expressed as percent relative to the mean baseline value.

\section{EVALUATION OF GLUTAMATE UPTAKE}

Extracellular glutamate levels were measured by a fluorimetric method using the Amplex Red Glutamic Acid assay kit (Invitrogen) as described previously with some modifications (Sato et al., 2003; Pawlak et al., 2005). After 3 h of OGD astrocyte culture medium was replaced by Hepes buffer containing $25 \mathrm{mM}$ glucose and $500 \mu \mathrm{M}$ glutamate. At each time point, $50 \mu \mathrm{l}$ of supernatants was transferred into 96-well microplates, and then mixed with $50 \mu \mathrm{l}$ substrate mixture $(100 \mathrm{mM}$ Amplex Red, $0.25 \mathrm{U} / \mathrm{ml}$ horseradish peroxidase, $0.08 \mathrm{U} / \mathrm{ml} \mathrm{L}$-glutamate oxidase, $0.5 \mathrm{U} / \mathrm{ml}$ glutamate pyruvate transaminase, and $200 \mu \mathrm{l}$ alanine) and incubated at $37^{\circ} \mathrm{C}$ for $30 \mathrm{~min}$. Fluorescence was measured using an automated microplate reader at a wavelength of $530 \mathrm{~nm}$ (vs. reference wavelength of $590 \mathrm{~nm}$ ). Glutamate concentrations were calculated from the standard curve with known glutamate amounts.

\section{IMMUNOCYTOCHEMISTRY}

Immunofluorescence labeling was performed as described (Plachez et al., 2004). Cultured cells were fixed with $4 \%$ (w/v) paraformaldehyde for $20 \mathrm{~min}$ and then washed with phosphate buffered saline (PBS). The cells were permeablized with $0.1 \%$ Triton X-100 in PBS for $1 \mathrm{~h}$ at room temperature. After blocked with bovine serum albumin (BSA) for $30 \mathrm{~min}$, the cultures were incubated with primary antibody (GLT-1 1:100) in PBS containing $0.5 \%(\mathrm{w} / \mathrm{v}) \mathrm{BSA}$ and $0.1 \%$ triton $\mathrm{X}-100$ at $4^{\circ} \mathrm{C}$ overnight. After washed with PBS for three times, the cells were incubated with biotinylated anti-rabbit $\operatorname{IgG}(1: 200)$ for $2 \mathrm{~h}$, followed by streptavidin-cy3(1:1000) for $2 \mathrm{~h}$. Hoechst 33342(1 $\mathrm{gg} / \mathrm{ml})$ was used for counterstain. The immunostaining images were observed under a fluorescence microscope (Leica, Germany). 


\section{REVERSE TRANSCRIPTION-POLYMERASE CHAIN REACTION}

Brain tissues were collected at $24 \mathrm{~h}$ following MCAO. RT-PCR was performed as described (Pawlak et al., 2005). Total RNA of brain tissues was extracted using Trizol reagent (Invitrogen Life Technologies, Carlsbad, CA, USA) according to the manufacturer's instructions. $3 \mu \mathrm{g}$ of total RNA was used for reverse transcription using Revert Aid ${ }^{\mathrm{TM}}$ First Strand cDNA Synthesis Kit (Fermentas, Burlington, ON, Canada). The PCR amplification was carried out as follows: a $30 \mathrm{~min}$ incubation at $58^{\circ} \mathrm{C}$ for cDNA synthesis, a 3 min hot start at $94^{\circ} \mathrm{C}$ followed by 32 cycles of denaturation at $94^{\circ} \mathrm{C}$ for $30 \mathrm{~s}$, annealing at $56^{\circ} \mathrm{C}$ for $30 \mathrm{~s}$, and extension at $72^{\circ} \mathrm{C}$ for $30 \mathrm{~s}$ with a final extension at $72^{\circ} \mathrm{C}$ for $5 \mathrm{~min}$. The amplified products were electrophoretically separated by $1.5 \%$ agarose gels containing ethidium bromide. Data were normalized to $\beta$-actin expression. Forward/reverse primers were:

GLT-1 5'-CAAGCTGATGGTGGAGTTCTT-3'/5'-CACGCTTG TCAATCCCTAGAT- $3^{\prime} ; \beta$-actin $5^{\prime}$-TGTGGCATCCATGAAACTAC A-3'/5'-CCACCAATCCACACAGAGTAC-3' .

\section{WESTERN BLOT}

Tissue of interest was homogenized on ice in the RIPA lysis buffer (Beyotime, China) containing $0.5 \mathrm{mM}$ phenylmethylsulfonyl fluoride (PMSF). Western blot was performed according to Ye etal. (2011b). Briefly, protein samples were electrophoresed on a $10 \%$ SDS-PAGE and subsequently transferred to polyvinylidene difluoride (PVDF) membrane (Millipore, Billerica, MA, USA). The membrane was incubated in blocking buffer containing 5\% non-fat dried milk at room temperature for $1 \mathrm{~h}$ and then probed with the primary antibody [GLT-1 at 1:1000; p-AKT at 1:500; p-ERK at 1:500; ERK at 1:1000; AKT at 1:1000; and glyceraldehyde 3-phosphate dehydrogenase (GAPDH) at 1:2000] in blocking buffer at $4^{\circ} \mathrm{C}$ overnight. The membrane was washed three times with TBST [tris-buffered saline (TBS) and 0.1\% Tween 20] and then incubated with horseradish peroxidase (HRP)-conjugated secondary antibody at room temperature for another $1 \mathrm{~h}$. Specific signals of proteins were visualized by chemiluminescence using the enhanced chemiluminescence (ECL) western blotting detection system (GE Healthcare, UK). For quantitative analysis, the ratio of the specific signals of protein (relative intensity of the signal) to that of GAPDH protein was calculated.

\section{STATISTICAL ANALYSIS}

All results were presented as mean with standard error mean (SEM). Microdialysis and glutamate uptake results were analyzed with repeated-measures analysis of variance, followed by Tukey HSD post hoc. Other results were analyzed using one-way ANOVA followed by Tukey HSD post test for multiple comparisons. Originpro 8 software was used for statistical tests. Statistical significance was established at $p<0.05$.

\section{RESULTS}

\section{Rd PROMOTES GLUTAMATE CLEARANCE IN VITRO AND IN VIVO}

To determine the effects of $\mathrm{Rd}$ on extracellular glutamate metabolism in vivo, we measured concentrations of extracellular glutamate during the period of $1.5 \mathrm{~h}$ ischemia and $2.5 \mathrm{~h}$ reperfusion in the ischemic striatum. As shown in Figure 1A, MCAO induced a rapid and marked increase in levels of extracellular glutamate, which reached the maximum at 60-100 min after stroke onset. The concentration of glutamate decreased after reperfusion but did not return to the basal lines during the measurement period in MCAO group. Compared with MCAO group, Rd administration $1 \mathrm{~h}$ before MCAO attenuated glutamate burst and promoted its recovery to the baseline $150 \mathrm{~min}$ after reperfusion. The differences in glutamate levels between MCAO and Rd-treated rats were significant at 100, 120, 140, and $160 \mathrm{~min}$ of occlusion (Figure 1A).

Furthermore, the effect of Rd on astrocyte glutamate uptake was evaluated by the quantification of the clearance of exogenous glutamate $(500 \mu \mathrm{M})$ from the astrocytes culture medium. Following $3 \mathrm{~h}$ of OGD insult, extracellular glutamate levels in the medium from cultured astrocytes with or without Rd pre-treatment (1, 10 , and $50 \mu \mathrm{M}, 12 \mathrm{~h}$ before OGD) were examined at different
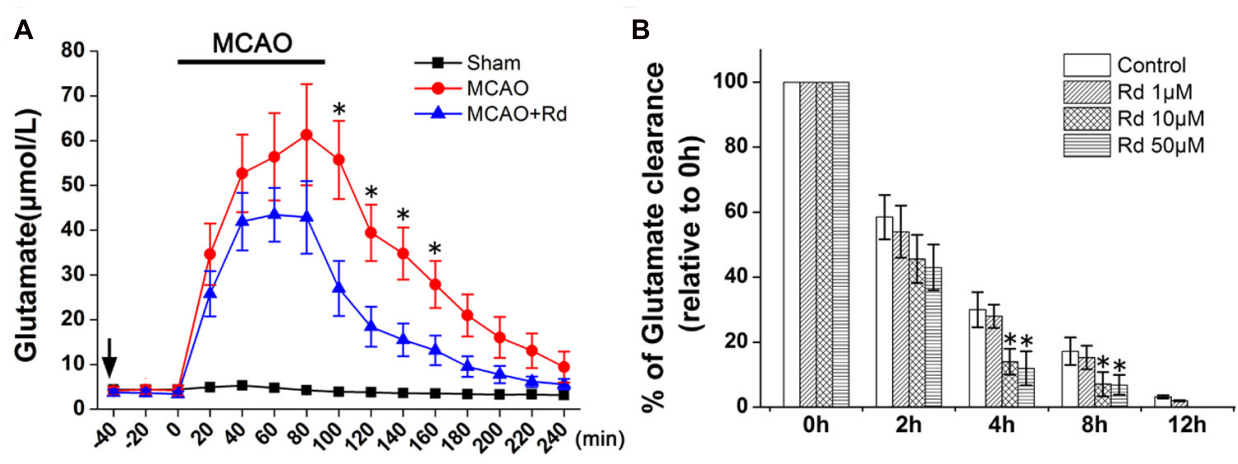

FIGURE 1 | Effect of Rd treatment on the extracellular concentrations of glutamate (A) Time course of the glutamate concentration determined with microdialysis in MCAO rats $(n=6)$, MACO rats pre-treated with $\mathrm{Rd}$ (depicted as black arrow, $30 \mathrm{mg} / \mathrm{kg}, n=8)$ or sham-operated rats $(n=7)$ subjected to 90-min MCAO (depicted as the black bar) followed by 150-min reperfusion. Values are the means \pm SEM. ${ }^{*} p<0.05$ vs. MCAO+Rd group. (B) Glutamate uptake was measured by indirectly quantifying the clearance of extracellular glutamate added. After $3 \mathrm{~h}$ of $\mathrm{OGD}, 500 \mu \mathrm{M}$ glutamate was added to culture medium. Glutamate concentrations were measured at different time points as indicated after OGD washout. Data are expressed as mean \pm SEM $(n=6)$. Mean values of $500 \mu \mathrm{M}$ glutamate were scaled to $100 \% .^{*} p<0.05$ vs. control group. 

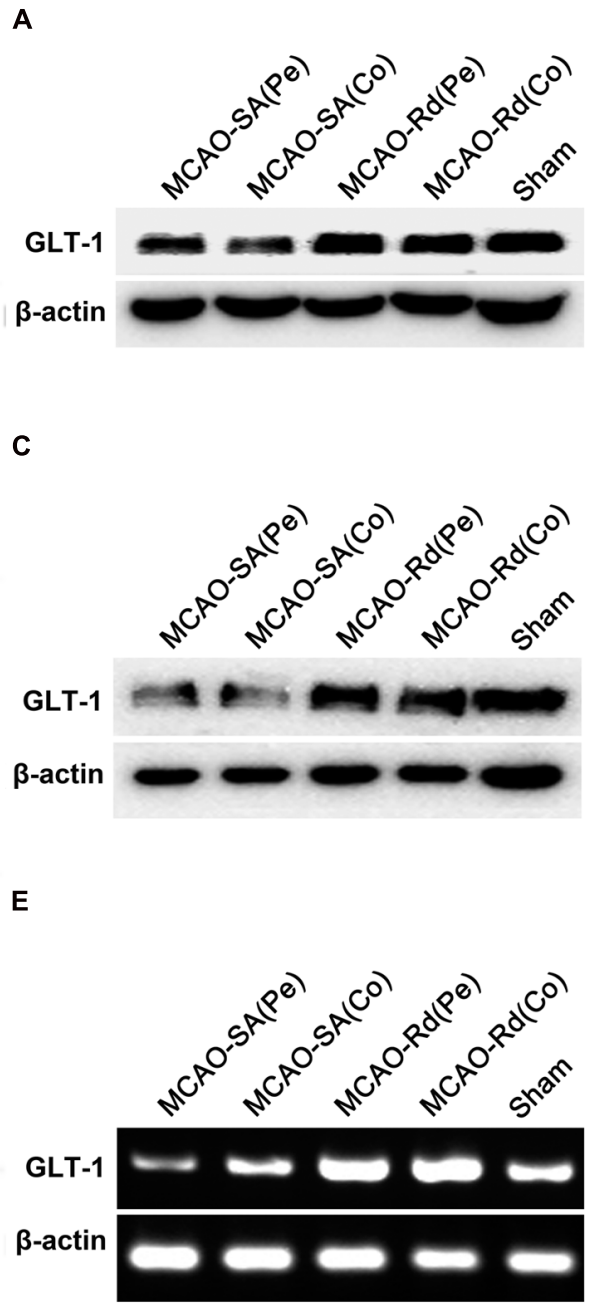

FIGURE 2 | Effect of Rd on GLT-1 expression in the core (Co) and penumbra (Pe) of ischemic brains. Western blotting analysis of GLT-1 expression at $4 \mathrm{~h}(\mathbf{A}, \mathbf{B})$ and $24 \mathrm{~h}(\mathbf{C}, \mathbf{D})$ following rat MCAO. (E,F) Analysis of

time intervals. 10 and $50 \mu \mathrm{M}$ Rd significantly enhanced the glutamate uptake in astrocyte cultures, compared with the control (Figure 1B).

\section{Rd UP-REGULATES GLT-1 EXPRESSION AFTER MACO}

Since GLT-1 is one of main transporters for removal of extracellular glutamate accumulation, we next investigated whether Rd could affect GLT-1 expression during and after rat MCAO (Figure 2). Western blotting results showed that GLT-1 protein levels were reduced 4 and $24 \mathrm{~h}$ after MCAO in both core and penumbra of ischemic rat brains, consistent with previous reports (Chen et al., 2005; Han et al., 2008). Rd treatment up-regulated GLT-1 expression at both time points after rat MCAO (Figures 2A-D). Similarly, RT-PCR analysis also showed an increase in mRNA expression levels of GLT-1 in the presence
D

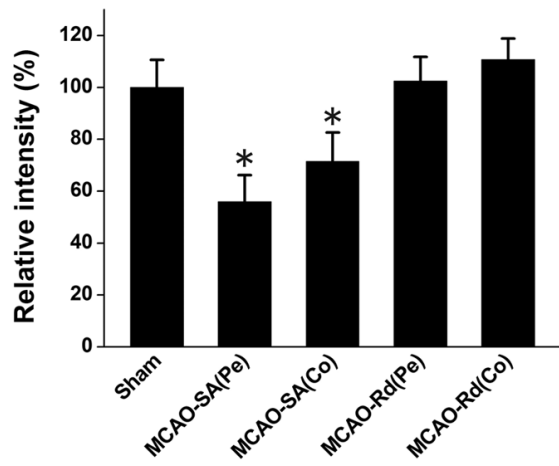

$\mathbf{F}$
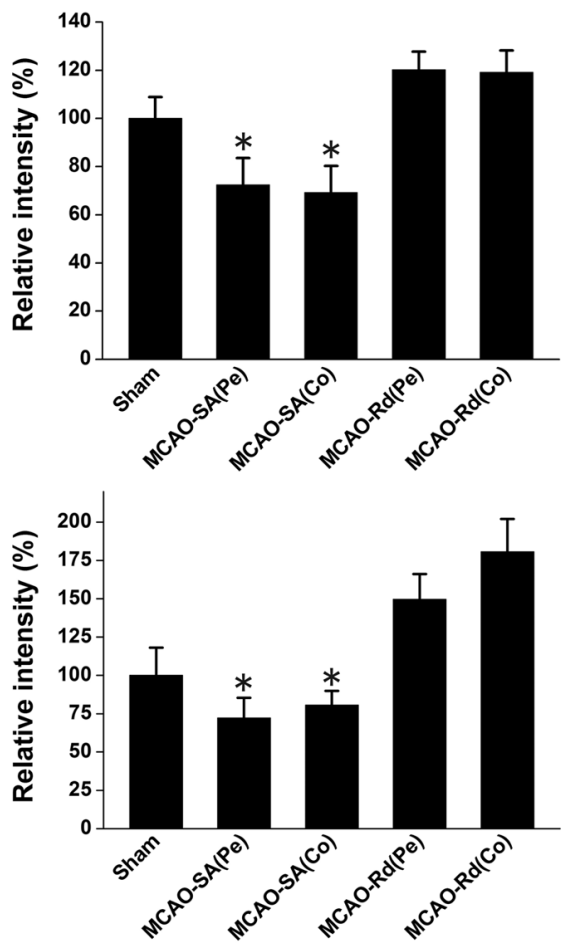

relative GLT-1 mRNA expression by semi-quantitative RT-PCR $24 \mathrm{~h}$ after rat MCAO. Data are expressed as mean $\pm \operatorname{SEM}(n=9)$. Mean values in sham-treated groups were scaled to $100 \% .{ }^{*} p<0.05$ vs. Rd-treated group. of Rd $24 \mathrm{~h}$ after MACO (Figures 2E,F), suggesting that Rd up-regulates GLT-1 expression after MACO.

\section{Rd INCREASES THE LEVELS OFPHOSPHO-PI3K/AKT AND PHOSPHO-ERK1/2}

PI3K/AKT and ERK mediate crucial regulatory pathways of cell survival (Kumar etal., 2004; Liu etal., 2012a). Both pathways are known to induce GLT-1 expression (Li et al., 2006; Lee et al., 2012a). We then explored whether Rd-induced GLT-1 up-regulation was mediated through PI3K/AKT and/or ERK pathway in astrocyte culture subjected to OGD insult. Western blotting showed that the levels of phospho-AKT and phospho-ERK were reduced 6 and $12 \mathrm{~h}$ after OGD, consistent with previous reports (Liu et al., 2012a; Xie et al., 2012; Xu et al., 2012). After the treatment of Rd, the levels of phosphorylation of AKT and ERK were 


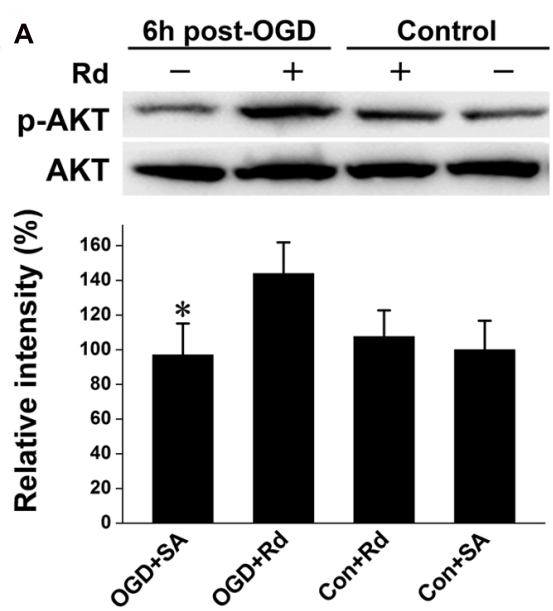

C
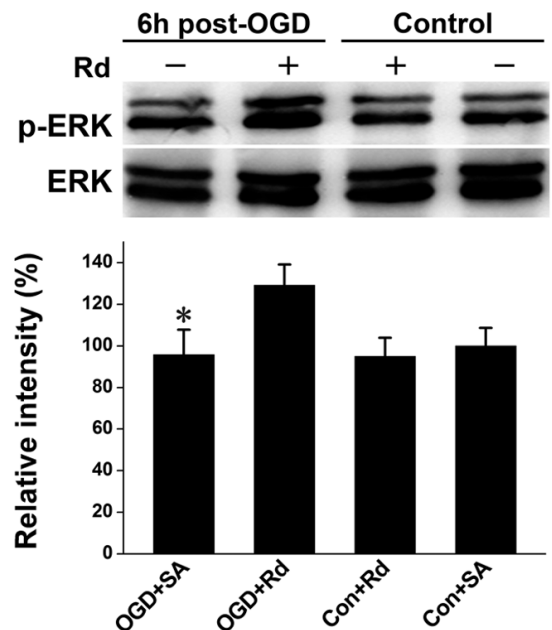

FIGURE 3 | Effect of Rd on phosphorylation of AKT and ERK1/2. Cultured astrocytes were harvested 6 and $12 \mathrm{~h}$ after OGD. Western blotting analysis of proteins extracted from astrocytes was performed using antibodies against p-AKT/AKT (A: $6 \mathrm{~h}$ and $\mathbf{B}: 12 \mathrm{~h}$ ) or $p$-ERK1/2

markedly enhanced (Figure 3), indicating that Rd can activate PI3K/AKT and ERK pathways.

\section{PI3K/AKT AND ERK1/2 PATHWAYS ARE INVOLVED IN Rd ENHANCED GLT-1 EXPRESSION AND GLUTAMATE UPTAKE}

Finally, we investigated whether Rd-induced GLT-1 up-regulation and glutamate uptake enhancement was mediated by PI3K/AKT and ERK1/2 pathways by using PI3K/AKT pathway inhibitor LY294002 or ERK1/2 pathway inhibitor PD98059. Western blotting and immunofluorescence labeling results showed that GLT-1 protein levels in cultured astrocytes decreased $12 \mathrm{~h}$ after OGD (Figure 4A). Similar to MCAO results, Rd increased GLT-1 expression $6 \mathrm{~h}$ (data not shown) and $12 \mathrm{~h}$ after OGD. While pre-treatment with LY294002 or PD98059 inhibited this effects of Rd (Figure 4B). Next, we tested the role of PI3K/AKT and ERK in Rd-induced glutamate uptake. As expected, Rd promoted astrocyte glutamate clearance, which can be abolished by co-treatment with LY294002

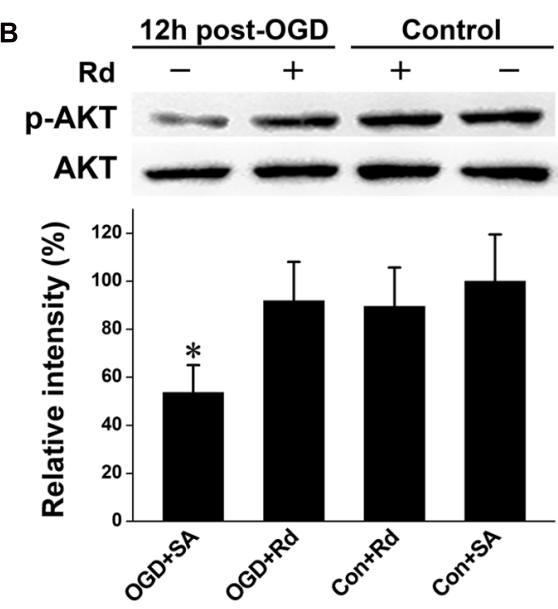

D
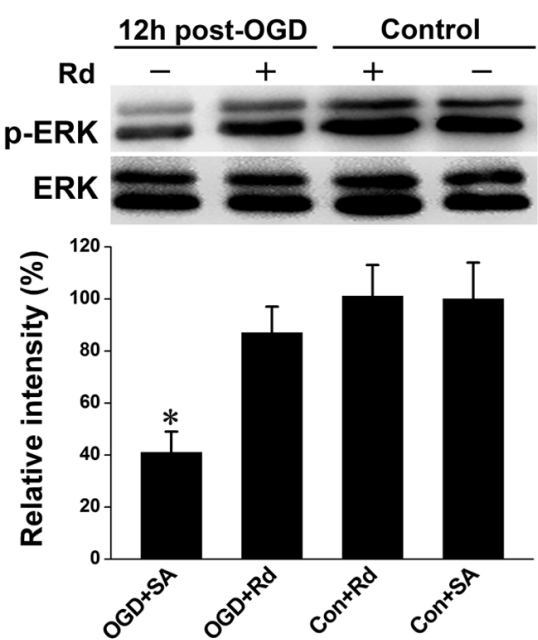

(C: $6 \mathrm{~h}$ and D: $12 \mathrm{~h}$ ) as described in Section "Materials and Methods." Data are expressed as mean $\pm \operatorname{SEM}(n=6)$. Mean values in sham-treated groups were scaled to $100 \%$. ${ }^{*} p<0.05$ vs. OGD + Rd-treated group.

or PD98059 (Figure 4C). Taken together, these results suggest that Rd may promote GLT-1 expression and glutamate uptake through $\mathrm{PI} 3 \mathrm{~K} / \mathrm{AKT}$ and ERK1/2 pathways.

\section{DISCUSSION}

In the present study, we investigated the effects of $\mathrm{Rd}$ on the expression of the astrocytic glutamate transporter GLT-1 as well as glutamate uptake activity in cultured astrocytes and MCAO rat. The results showed that Rd increased mRNA and protein levels of GLT-1. This effects can be inhibited by the AKT or ERK1/2 inhibitor, suggesting that AKT and ERK1/2 signaling pathways are involved in Rd-mediated effects on extracellular glutamate metabolism.

Glutamate-induced excitotoxicity is an important factor responsible for cell death in many central nervous system (CNS) disorders (Benveniste et al., 1984). Excessive extracellular glutamate over-stimulates ionotropic GluRs, such as NMDA, 

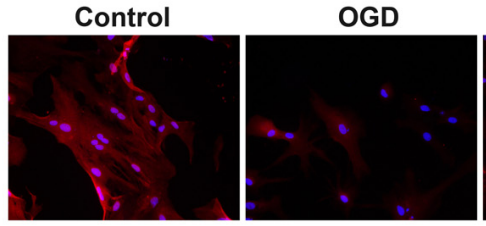

B

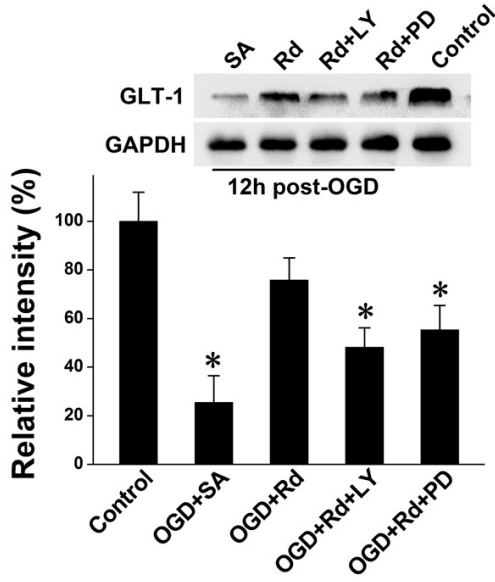

FIGURE 4 | PI3K/AKT and ERK are involved in Rd-induced GLT-1 up-regulation and glutamate uptake enhancement. $20 \mu \mathrm{M}$ PD98059 (PD) or $5 \mu \mathrm{M}$ LY294002 (LY) was pre-treated $12 \mathrm{~h}$ before OGD. (A) Double immunofluorescence labeling (GLT-Red, Hoechst-blue) $12 \mathrm{~h}$ after OGD. (B) Western blotting results $12 \mathrm{~h}$ after OGD. (C) Glutamate uptake assays. After

C

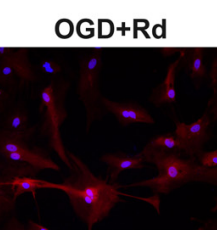

OGD+Rd+LY
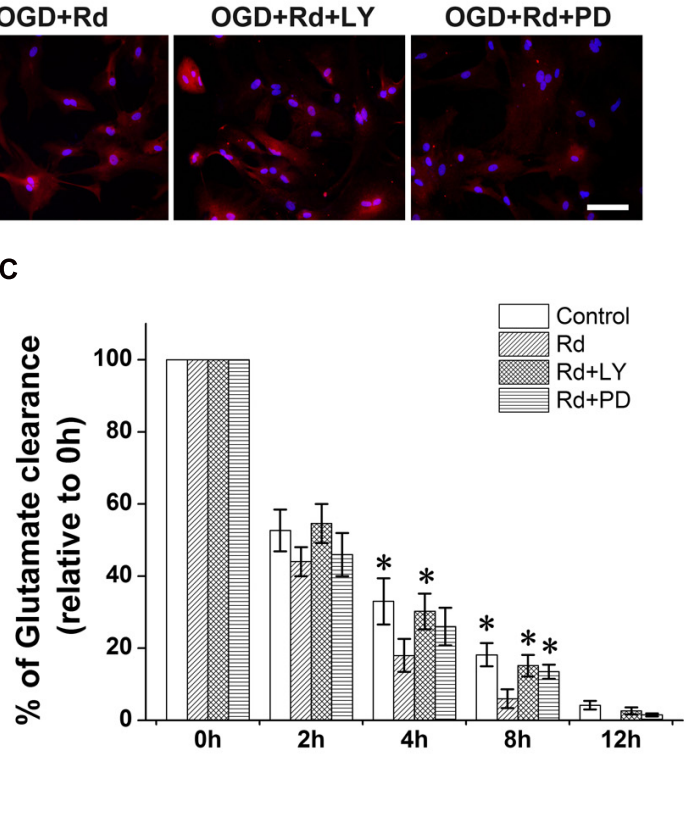

$3 \mathrm{~h}$ of OGD, $500 \mu \mathrm{M}$ glutamate was added to medium, and glutamate concentrations were measured at different time intervals (from 2 to $12 \mathrm{~h}$ after OGD washout). Data are expressed as mean $\pm \operatorname{SEM}(n=6)$. Mean values in sham-treated groups were scaled to $100 \% .{ }^{*} p<0.05$ vs. OGD + Rd group subsequently initiating a series of downstream lethal events including oxidative stress, mitochondrial dysfunction, and inflammation (Szydlowska and Tymianski, 2010). Astrocytes play a crucial role in the removal of extracellular glutamate to prevent against glutamate excitotoxicity (Rothstein et al., 1996). Two types of glutamate transporters, GALST and GLT-1, are identified in astrocytes: GLAST is predominantly expressed in the cerebellum and required to keep extracellular glutamate at a physiological level (Watase et al., 1998). GLT-1 is abundant in various area of the forebrain, including hippocampus, cerebral cortex, and the striatum. It is responsible for more than $90 \%$ of glutamate uptake in these areas (Danbolt, 2001). A group of studies have showed that GLT-1 is required for uptake of extracellular glutamate to protect neurons under ischemic conditions. For example, knockdown of GLT-1 expression exacerbated ischemia-induced neuronal damage and causes enlarged infarct volume (Rao et al., 2001). In contrast, targeted overexpression of GLT-1 decreased the glutamate overflow and reduced the cellular and behavioral deficits induced by ischemic stroke (Harvey et al., 2011). Thus, GLT-1 plays a neuronal protective role during ischemic stroke. Several studies showed decreases in GLT-1 mRNA and protein levels in the rat brain following ischemic insult. In addition, reduction of GLT-1 expression was observed $6 \mathrm{~h}$ after cerebral ischemia and was in part causative of glutamate-induced neurotoxicity in early phases of cerebral ischemia (Yeh et al., 2005). In our study, administration of Rd significantly increased the expression of GLT-1 mRNA and protein levels. These results suggest that Rd alleviates excitatory toxicity at least in part by restoring the expression of GLT-1.

Additionally, GLT-1 is also proposed to release intracellular glutamate under ischemic conditions (Phillis et al., 2000). However, no effect of dihydrokainate, a blocker of the reversed GLT-1 uptake, on ischemia-induced glutamate release was observed (Roettger and Lipton, 1996). Glutamate release in less severely ischemic brain was shown to occur mainly via volume-activated anion channels but not via GLT-1 reversal (Feustel et al., 2004). These studies suggest that GLT-1 reversal may not be an essential mechanism for increased extracellular glutamate concentration, particularly in ischemic penumbra.

As critical mediators, AKT and ERK1/2 signaling pathways are involved in cell proliferation, differentiation, and adaptation (Kumar et al., 2004; Liu et al., 2012a). Phosphorylation of ERK1/2 and AKT activates the transcription factors, NF-kB, and cAMPresponse element binding protein (CREB), which in turn control the transcription of GLT-1(Li et al., 2006; Lee et al., 2012a). In addition, other types of ginsenosides, such as Rb1, Rg1, have been reported to affect AKT and ERK1/2 pathways (Lan et al., 2011; Hashimoto et al., 2012; Yan et al., 2013). Our present study further showed that Rd activated AKT and ERK1/2 signaling pathways (Figure 4), subsequently affecting GLT-1 expression and extracellular glutamate uptake.

Estrogen receptor (ER)-mediated PI3K/AKT and ERK1/2 activation are well-documented in protection against cell death. Recently, several reports showed that ER activation increased GLT-1 expression and glutamate uptake (Lee etal., 2012a,b). 
Ginseng has been recommended for alleviation of the symptoms of menopause, indicating that some components of ginseng act as phytoestrogen involve activation of the ER (Amato et al., 2002). Some evidence showed that ginseng extracts were able to stimulate the growth of ER-positive cells (Duda etal., 1999). Ginsenosides were structurally and functionally similar to $17 \beta$-estradiol (Lee et al., 2003; Lau etal., 2008) and activated AKT and ERK pathways (Lan et al., 2011; Hashimoto etal., 2012; Yan et al., 2013). Thus, we proposed that acting on ER may account for Rd-induced AKT and ERK1/2 activation and sequential GLT-1 up-regulation. Yet, Rd may also affect the functions of GLT-1 in several ways, including cell trafficking, splicing, and posttranslational modification. Thus, the possible links between Rd and ER still require further clarification.

In summary, in the present study we showed that Rd could promote extracellular glutamate clearance by up-regulating GLT-1 expression through PI3K/AKT and ERK1/2 pathways.

\section{ACKNOWLEDGMENTS}

The authors are grateful to Ms. Dongyun Feng for technical assistance. This work was supported by grants from Science and technology project of Shaanxi Province (2013k12-01-02).

\section{AUTHOR CONTRIBUTIONS}

Xiao Zhang: most of the research work and paper writing. Ming Shi: part of the paper writing and experiment design. Magnar Bjørås: polishing manuscript, great assistance in main research project design. Wei Wang: paper revision, great assistance in experiment design. Guangyun Zhang: funds support. Junliang Han: great assistance in Microdialysis experiment. Zhirong Liu: great assistance in experiment design. Yunxia Zhang: doing part of the Western blot experiment. Bing Wang: doing part of the Immunocytochemistry experiment. Jing Chen: glial cultures and OGD experiment. Yi Zhu: doing the animal surgery. Lize Xiong: main research project design. Gang Zhao: main research project and experiment design.

\section{REFERENCES}

Amato, P., Christophe, S., and Mellon, P. L. (2002). Estrogenic activity of herbs commonly used as remedies for menopausal symptoms. Menopause 9, 145-150. doi: 10.1097/00042192-200203000-00010

Benveniste, H., Drejer, J., Schousboe, A., and Diemer, N. H. (1984). Elevation of the extracellular concentrations of glutamate and aspartate in rat hippocampus during transient cerebral ischemia monitored by intracerebral microdialysis. $J$. Neurochem. 43, 1369-1374. doi: 10.1111/j.1471-4159.1984.tb05396.x

Chen, J. C., Hsu-Chou, H., Lu, J. L., Chiang, Y. C., Huang, H. M., Wang, H. L., et al. (2005). Down-regulation of the glial glutamate transporter GLT-1 in rat hippocampus and striatum and its modulation by a group III metabotropic glutamate receptor antagonist following transient global forebrain ischemia. Neuropharmacology 49, 703-714. doi: 10.1016/j.neuropharm.2005.05.002

Danbolt, N. C. (2001). Glutamate uptake. Prog. Neurobiol. 65, 1-105. doi: 10.1016/S0301-0082(00)00067-8

Duda, R. B., Zhong, Y., Navas, V., Li, M. Z., Toy, B. R., and Alavarez, J. G. (1999). American ginseng and breast cancer therapeutic agents synergistically inhibit MCF-7 breast cancer cell growth. J. Surg. Oncol. 72, 230-239. doi: 10.1002/(SICI) 1096-9098(199912)72:4<230::AID-JSO9>3.0.CO;2-2

Feustel, P. J., Jin, Y., and Kimelberg, H. K. (2004). Volume-regulated anion channels are the predominant contributors to release of excitatory amino acids in the ischemic cortical penumbra. Stroke 35, 1164-1168. doi: 10.1161/01.STR.0000124127.57946.a1
Gegelashvili, G., and Schousboe, A. (1997). High affinity glutamate transporters: regulation of expression and activity. Mol. Pharmacol. 52, 6-15.

Goldstein, L. B., Adams, R., Alberts, M. J., Appel, L. J., Brass, L. M., Bushnell, C. D., et al. (2006). Primary prevention of ischemic stroke: a guideline from the American heart association/American stroke association atroke council: cosponsored by the atherosclerotic peripheral vascular disease interdisciplinary working group; cardiovascular nursing council; clinical cardiology council; nutrition, physical activity, and metabolism council; and the quality of care and outcomes research interdisciplinary working group. Circulation 113, e873-e923.

Gropen, T. I., Gagliano, P. J., Blake, C. A., Sacco, R. L., Kwiatkowski, T., Richmond, N. J., etal. (2006). Quality improvement in acute stroke: the New York State Stroke Center Designation Project. Neurology 67, 88-93. doi: 10.1212/01.wnl.0000223622.13641.6d

Han, F., Shioda, N., Moriguchi, S., Qin, Z. H., and Fukunaga, K. (2008). Downregulation of glutamate transporters is associated with elevation in extracellular glutamate concentration following rat microsphere embolism. Neurosci. Lett. 430, 275-280. doi: 10.1016/j.neulet.2007.11.021

Harvey, B. K., Airavaara, M., Hinzman, J., Wires, E. M., Chiocco, M. J., Howard, D. B., et al. (2011). Targeted over-expression of glutamate transporter 1 (GLT-1) reduces ischemic brain injury in a rat model of stroke. PLoS ONE 6:e22135. doi: 10.1371/journal.pone.0022135

Hashimoto, R., Yu, J., Koizumi, H., Ouchi, Y., and Okabe, T. (2012). Ginsenoside $\mathrm{Rb} 1$ prevents $\mathrm{MPP}(+)$-induced apoptosis in $\mathrm{PC1} 2$ cells by stimulating estrogen receptors with consequent activation of ERK1/2, Akt and inhibition of SAPK/JNK, p38 MAPK. Evid. Based Complement. Alternat. Med. 2012, 693717. doi: $10.1155 / 2012 / 693717$

Kramer, M., Dang, J., Baertling, F., Denecke, B., Clarner, T., Kirsch, C., et al. (2010). TTC staining of damaged brain areas after MCA occlusion in the rat does not constrict quantitative gene and protein analyses. J. Neurosci. Methods 187, 84-89. doi: 10.1016/j.jneumeth.2009.12.020

Kumar, P., Miller, A. I., and Polverini, P. J. (2004). p38 MAPK mediates gammairradiation-induced endothelial cell apoptosis, and vascular endothelial growth factor protects endothelial cells through the phosphoinositide 3-kinase-Akt-Bcl-2 pathway. J. Biol. Chem. 279, 43352-43360. doi: 10.1074/jbc.M405777200

Lan, T. H., Xu, Z. W., Wang, Z., Wu, Y. L., Wu, W. K., and Tan, H. M. (2011). Ginsenoside Rb1 prevents homocysteine-induced endothelial dysfunction via PI3K/Akt activation and PKC inhibition. Biochem. Pharmacol. 82, 148-155. doi: 10.1016/j.bcp.2011.04.001

Lau, W. S., Chan, R. Y., Guo, D. A., and Wong, M. S. (2008). Ginsenoside Rg1 exerts estrogen-like activities via ligand-independent activation of ERalpha pathway. J. Steroid Biochem. Mol. Biol. 108, 64-71. doi: 10.1016/j.jsbmb.2007.06.005

Lee, E., Sidoryk-Wegrzynowicz, M., Wang, N., Webb, A., Son, D. S., Lee, K., et al. (2012a). GPR30 regulates glutamate transporter GLT-1 expression in rat primary astrocytes. J. Biol. Chem. 287, 26817-26828. doi: 10.1074/jbc.M112. 341867

Lee, E., Sidoryk-Wegrzynowicz, M., Yin, Z., Webb, A., Son, D. S., and Aschner, M. (2012b). Transforming growth factor-alpha mediates estrogen-induced upregulation of glutamate transporter GLT-1 in rat primary astrocytes. Glia 60 , 1024-1036. doi: 10.1002/glia.22329

Lee, Y., Jin, Y., Lim, W., Ji, S., Choi, S., Jang, S., et al. (2003). A ginsenosideRh1, a component of ginseng saponin, activates estrogen receptor in human breast carcinoma MCF-7 cells. J. Steroid Biochem. Mol. Biol. 84, 463-468. doi: 10.1016/S0960-0760(03)00067-0

Li, L. B., Toan, S. V., Zelenaia, O., Watson, D. J., Wolfe, J. H., Rothstein, J. D., et al. (2006). Regulation of astrocytic glutamate transporter expression by Akt: evidence for a selective transcriptional effect on the GLT-1/EAAT2 subtype. J. Neurochem. 97, 759-771. doi: 10.1111/j.1471-4159.2006.03743.x

Li, X. Y., Liang, J., Tang, Y. B., Zhou, J. G., and Guan, Y. Y. (2010). Ginsenoside Rd prevents glutamate-induced apoptosis in rat cortical neurons. Clin. Exp. Pharmacol. Physiol. 37, 199-204. doi: 10.1111/j.1440-1681.2009.05286.x

Liu, R. L., Xiong, Q. J., Shu, Q., Wu, W. N., Cheng, J., Fu, H., et al. (2012a). Hyperoside protects cortical neurons from oxygen-glucose deprivation-reperfusion induced injury via nitric oxide signal pathway. Brain Res. 1469, 164-173. doi: 10.1016/j.brainres.2012.06.044

Liu, X., Wang, L., Wen, A., Yang, J., Yan, Y., Song, Y., et al. (2012b). Ginsenoside-Rd improves outcome of acute ischaemic stroke - a randomized, double-blind, placebo-controlled, multicenter trial. Eur. J. Neurol. 19, 855-863. doi: 10.1111/j.1468-1331.2011.03634.x 
Liu, X., Xia, J., Wang, L., Song, Y., Yang, J., Yan, Y., et al. (2009). Efficacy and safety of ginsenoside-Rd for acute ischaemic stroke: a randomized, double-blind, placebo-controlled, phase II multicenter trial. Eur. J. Neurol. 16, 569-575. doi: 10.1111/j.1468-1331.2009.02534.x

Lopez, M. V., Cuadrado, M. P., Ruiz-Poveda, O. M., Del Fresno, A. M., and Accame, M. E. (2007). Neuroprotective effect of individual ginsenosides on astrocytes primary culture. Biochim. Biophys. Acta 1770, 1308-1316. doi: 10.1016/j.bbagen.2007.06.008

Mitani, A., and Tanaka, K. (2003). Functional changes of glial glutamate transporter GLT-1 during ischemia: an in vivo study in the hippocampal CA1 of normal mice and mutant mice lacking GLT-1. J. Neurosci. 23, 7176-7182.

Pawlak, J., Brito, V., Kuppers, E., and Beyer, C. (2005). Regulation of glutamate transporter GLAST and GLT-1 expression in astrocytes by estrogen. Brain Res. Mol. Brain Res. 138, 1-7. doi: 10.1016/j.molbrainres.2004.10.043

Phillis, J. W., Ren, J., and O’Regan, M. H. (2000). Transporter reversal as a mechanism of glutamate release from the ischemic rat cerebral cortex: studies with DL-threo-beta-benzyloxyaspartate. Brain Res. 880, 224. doi: 10.1016/S00068993(00)02755-4

Plachez, C., Martin, A., Guiramand, J., and Recasens, M. (2004). Astrocytes repress the neuronal expression of GLAST and GLT glutamate transporters in cultured hippocampal neurons from embryonic rats. Neurochem. Int. 45, 1113-1123. doi: 10.1016/j.neuint.2004.03.030

Radad, K., Gille, G., Liu, L., and Rausch, W. D. (2006). Use of ginseng in medicine with emphasis on neurodegenerative disorders. J. Pharmacol. Sci. 100, 175-186. doi: 10.1254/jphs.CRJ05010X

Rao, V. L., Dogan, A., Todd, K. G., Bowen, K. K., Kim, B. T., Rothstein, J. D., et al. (2001). Antisense knockdown of the glial glutamate transporter GLT-1, but not the neuronal glutamate transporter EAAC1, exacerbates transient focal cerebral ischemia-induced neuronal damage in rat brain. J. Neurosci. 21, 18761883.

Roettger, V., and Lipton, P. (1996). Mechanism of glutamate release from rat hippocampal slices during in vitro ischemia. Neuroscience 75, 677-685. doi: 10.1016/0306-4522(96)00314-4

Rothstein, J. D., Dykes-Hoberg, M., Pardo, C. A., Bristol, L. A., Jin, L., Kuncl, R. W., et al. (1996). Knockout of glutamate transporters reveals a major role for astroglial transport in excitotoxicity and clearance of glutamate. Neuron 16, 675-686. doi: 10.1016/S0896-6273(00)80086-0

Sato, K., Matsuki, N., Ohno, Y., and Nakazawa, K. (2003). Estrogens inhibit l-glutamate uptake activity of astrocytes via membrane estrogen receptor alpha. J. Neurochem. 86, 1498-1505. doi: 10.1046/j.1471-4159.2003. 01953.x

Szydlowska, K., and Tymianski, M. (2010). Calcium, ischemia and excitotoxicity. Cell Calcium 47, 122-129. doi: 10.1016/j.ceca.2010.01.003

Van der Schyf, C. J., Geldenhuys, W. J., and Youdim, M. B. (2006). Multifunctional drugs with different CNS targets for neuropsychiatric disorders. J. Neurochem. 99, 1033-1048. doi: 10.1111/j.1471-4159.2006.04141.x

Watase, K., Hashimoto, K., Kano, M., Yamada, K., Watanabe, M., Inoue, Y., et al (1998). Motor discoordination and increased susceptibility to cerebellar injury in GLAST mutant mice. Eur. J. Neurosci. 10, 976-988. doi: 10.1046/j.14609568.1998.00108.x
Xie, R., Li, X., Ling, Y., Shen, C., Wu, X., Xu, W., et al. (2012). Alpha-lipoic acid pre- and post-treatments provide protection against in vitro ischemia-reperfusion injury in cerebral endothelial cells via Akt/mTOR signaling. Brain Res. 1482, 81-90. doi: 10.1016/j.brainres.2012. 09.009

Xu, C., Lv, L., Zheng, G., Li, B., Gao, L., and Sun, Y. (2012). Neuregulin1beta1 protects oligodendrocyte progenitor cells from oxygen glucose deprivation injury induced apoptosis via ErbB4-dependent activation of PI3-kinase/Akt. Brain Res. 1467, 104-112. doi: 10.1016/j.brainres.2012.05.044

Yan, J., Liu, Q., Dou, Y., Hsieh, Y., Liu, Y., Tao, R., et al. (2013). Activating glucocorticoid receptor-ERK signaling pathway contributes to ginsenoside Rg1 protection against beta-amyloid peptide-induced human endothelial cells apoptosis. J. Ethnopharmacol. 147, 456-466. doi: 10.1016/j.jep.2013. 03.039

Ye, R., Li, N., Han, J., Kong, X., Cao, R., Rao, Z., et al. (2009). Neuroprotective effects of ginsenoside Rd against oxygen-glucose deprivation in cultured hippocampal neurons. Neurosci. Res. 64, 306-310. doi: 10.1016/j.neures.2009. 03.016

Ye, R., Yang, Q., Kong, X., Han, J., Zhang, X., Zhang, Y., et al. (2011a). Ginsenoside $\mathrm{Rd}$ attenuates early oxidative damage and sequential inflammatory response after transient focal ischemia in rats. Neurochem. Int. 58, 391-398. doi: 10.1016/j.neuint.2010.12.015

Ye, R., Zhang, X., Kong, X., Han, J., Yang, Q., Zhang, Y., et al. (2011b). Ginsenoside $\mathrm{Rd}$ attenuates mitochondrial dysfunction and sequential apoptosis after transient focal ischemia. Neuroscience 178, 169-180. doi: 10.1016/j.neuroscience.2011. 01.007

Yeh, T. H., Hwang, H. M., Chen, J. J., Wu, T., Li, A. H., and Wang, H. L. (2005). Glutamate transporter function of rat hippocampal astrocytes is impaired following the global ischemia. Neurobiol. Dis. 18, 476-483. doi: 10.1016/j.nbd.2004. 12.01

Conflict of Interest Statement: The authors declare that the research was conducted in the absence of any commercial or financial relationships that could be construed as a potential conflict of interest.

Received: 27 September 2013; paper pending published: 29 October 2013; accepted: 24 November 2013; published online: 11 December 2013

Citation: Zhang X, Shi M, Bjørås M, Wang W, Zhang G, Han J, Liu Z, Zhang Y, Wang B, Chen J, Zhu Y, Xiong L and Zhao G (2013) Ginsenoside Rd promotes glutamate clearance by up-regulating glial glutamate transporter GLT-1 via PI3K/AKT and ERK1/2 pathways. Front. Pharmacol. 4:152. doi: 10.3389/fphar.2013.00152

This article was submitted to Cardiovascular and Smooth Muscle Pharmacology, a section of the journal Frontiers in Pharmacology.

Copyright (c) 2013 Zhang, Shi, Bjørås, Wang, Zhang, Han, Liu, Zhang, Wang, Chen, $\mathrm{Zhu}$, Xiong and Zhao. This is an open-access article distributed under the terms of the Creative Commons Attribution License (CC BY). The use, distribution or reproduction in other forums is permitted, provided the original author(s) or licensor are credited and that the original publication in this journal is cited, in accordance with accepted academic practice. No use, distribution or reproduction is permitted which does not comply with these terms. 